\title{
DEGRADATION OF SOLAR ARRAY COMPONENTS IN A COMBINED UV/VUV HIGH TEMPERATURE TEST ENVIRONMENT
}

\author{
Christel Nömayr $^{(1)}$, Claus Zimmermann ${ }^{(1)}$, Premysl Janik $^{(2)}$, Christopher Semprimoschnig $^{(2)}$ \\ (1) Airbus Defence and Space, 81663 Munich, Germany, Email: christel.anke.noemayr@airbus.com \\ (2) ESA/ESTEC, Keplerlaan 1, 2200AG Noordwijk, The Netherlands
}

\begin{abstract}
BepiColombo is the joint mission of the European Space Agency (ESA) and the Japanese Aerospace Exploration Agency (JAXA) to explore the planet mercury. The European contributions, namely the mercury transfer module (MTM) and the mercury planetary orbiter (MPO), are both powered by deployable solar arrays. Many materials and technologies are at their limit under the harsh highintensity, high-temperature (HIHT) conditions of the mission. Synergistic effects like photo fixation and photo enhanced contamination by ultra violet and vacuum ultra violet radiation (UV/VUV) on sunlit surfaces are considered to play an important role in the HIHT environment of the BepiColombo mission.

A design verification test under UV/VUV conditions of sun exposed materials and technologies on component level is presented which forms part of the overall verification and qualification of the solar array design of the MTM and MPO. The test concentrates on the selfcontamination aspects and the resulting performance losses of the solar array under high intensity and elevated temperature environment representative for the photovoltaic assembly (PVA).
\end{abstract}

\section{INTRODUCTION}

A classical approach to predict contamination levels is based on the surface temperature in comparison to the temperature of the outgassing source. Especially on very hot surfaces contamination would then not be an issue since any outgassing molecule would not stick to the surface at all or would not have a sufficiently long residence time for the build-up of a contamination layer. However, laboratory as well as in-orbit experiments [1] have shown that when sunlit, warmer surfaces become more degraded by contamination than they would have been expected by a pure assessment of their surface temperature. Also the observed degradation of a GPS solar array might be explained to be caused partially by photo enhanced contamination effects [2].

The exact mechanisms that play a role for contamination and degradation of UV/VUV irradiated surfaces are complicated. One explanation for the formation of non-volatile contamination layers is that the adsorbed molecules polymerize to species with longer residence times (= photo fixation). Another mechanism discussed is the photochemical modification of a molecule immediately before it is sticking to the surface leading to a different sticking behaviour (= photo enhanced deposition). The exact photochemistry and the interplay between outgassing molecule arrival rate and photonic density are complicating the phenomenon when setting up a model to calculate expected contamination levels.

Besides the formation of the contamination layer itself, the photochemical development of the contamination film over time, typically characterized by a darkening, has to be considered. One important consequence of this behaviour for solar arrays is the decrease of transmission of the cover glasses which translates into solar cell performance loss. Another important consequence is the higher absorbance of thermal control materials e.g. OSRs which has an impact on the overall solar array temperature. The photochemistry of the representative contamination mixture would have to be understood in detail in order to analyse the degradation by modelling.

All the aforementioned aspects and difficulties made it necessary to identify an alternative experimental approach to assess the impact of combined outgassing and UV/VUV under relevant mission conditions on the BepiColombo solar array. The idea was to test the UV/VUV effect in combination with outgassing as close as possible to the final application. The test plan therefore tried to mimic not only the environmental conditions as far as possible in an on-ground based simulation, but also the most relevant material constellations and solar array outgassing sources.

\section{DESIGN OF TEST COUPON}

The focus of the test was on the materials and technologies of the PVA [3]: solar cell with cover glass, OSRs, Kapton VDA (vapour deposited aluminium), panel edge shield coatings, and cable bundle sleeves. All of the items have a direct or indirect impact on the solar array performance by power generation and thermal control. In parallel the main outgassing contributors which can be considered for the outgassing environment of these items were included in the sample design: Cyanate ester face skins and film adhesives, RTV (room temperature vulcanizing) silicones, and Kapton foil material. 
There were several constraints to be considered for the design of the test coupon. Due to the test conditions and the uniqueness of facilities that can create them, the available test space was limited to $200 \mathrm{~mm}$ x $200 \mathrm{~mm}$.

Another difficulty was to ensure thermal control during the test. Typical solar array coupon designs contain representative to the flight design, a piece of $\mathrm{CFC}$ (carbon fibre composite) faceskin / honeycomb core sandwich as a substrate. For a reliable temperature control in a vacuum test environment, however, the intimate contact of the samples to the facility base plate (which provides cooling and heating) is required. This is even more delicate with the heat input by UV/VUV illumination from top. The solution was to bond the solar cell assemblies (SCA), optical solar reflectors (OSRs), and Kapton VDA as well as a cable bundle sample to representative cyanate ester face skin material. The face skin was bonded directly onto Invar plates being screwed directly onto the sample plate. This is shown in the cross-sectional setup of an SCA sample in Fig. 1. The sample plate was then mounted to the facility plate which allowed the sample temperature to be controlled.
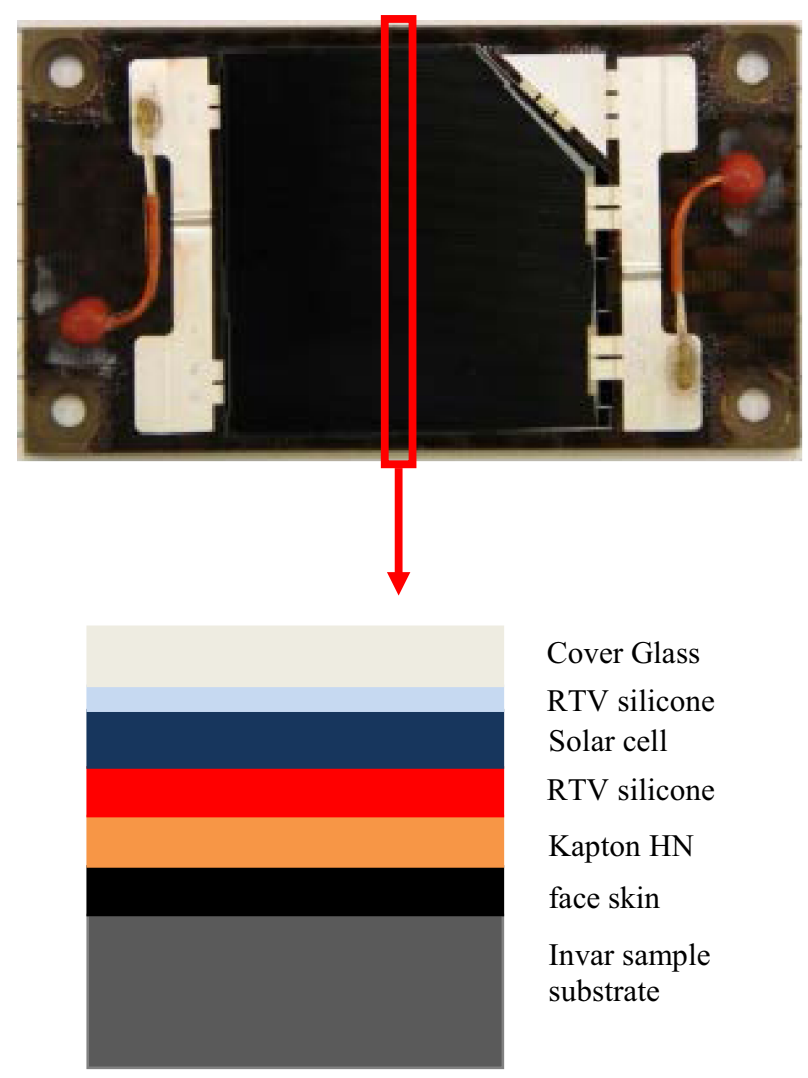

Cover Glass

RTV silicone

Solar cell

RTV silicone

Kapton HN

face skin

Invar sample substrate

Figure 1. SCA sample with schematic cross-section

In order to mimic the situation on the solar array panel, the test specimen were arranged as representative as possible on the sample base plate as shown in Fig. 2. It was not possible to implement all the view factors e.g. the $90^{\circ}$ orientation of the panel edge shields. Yet the tested condition represented part of the combination which can be assumed to represent an envelope in terms of degradation.

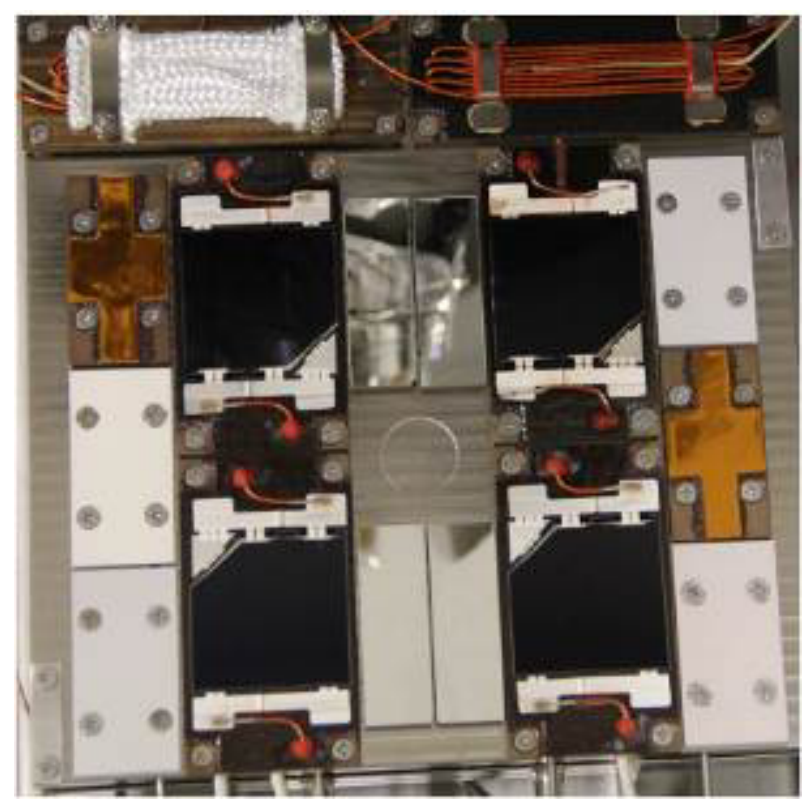

Figure 2. Test coupon arrangement on sample plate

In order to simulate the significantly higher temperature for the cable bundle with Nextel sleeve, which is part of the transfer harness as illustrated in Fig. 3, it was necessary to thermally isolate it by washers to the rest of the samples and induce additional heating by current injection through the cable bundle.

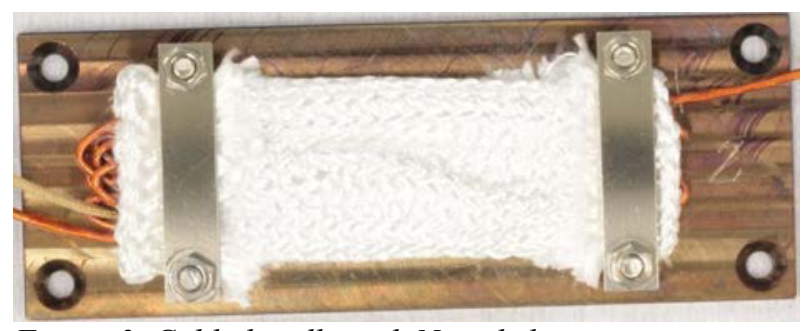

Figure 3. Cable bundle with Nextel sleeve

A second cable bundle without sleeve was kept at solar cell temperature, again with the help of current injection.

In order to test the required setting for the test temperatures a set of pre-test samples was used with embedded thermocouples to read out the obtained temperatures with a master thermocouple used as facility control reference during the test.

\section{EXPERIMENTAL}

The test was conducted in the CROSS1 vacuum facility at ESTEC. The facility offers exposure to ultraviolet (up to $12 \mathrm{SC}$ ) as well as vacuum ultraviolet. The base plate of the facility can be heated and cooled to control the 
sample temperature.

The UV intensity varied between 7 and 12 solar constants (SC) on the sample plate. The VUV intensity during each test phase varied due to the contamination of the lamp which is inside the chamber. Because of this fact, intermediate cleaning steps were undertaken. The measured VUV intensity itself was lower compared to the UV intensity. This was predominantly related to the aspect angle of $45^{\circ}$ of the VUV illumination. The measured values ranged from $<1$ to $9 \mathrm{SC}$.

The test temperatures for the SCA samples were set to $200^{\circ} \mathrm{C}$ for 2400 test hours (cycle $1-4$ ) and $215^{\circ} \mathrm{C}$ (cycle 5 ) for the last 600 test hours. The Nextel cable bundle was set to $275^{\circ} \mathrm{C}$ for 2400 test hours followed by a stepwise temperature increase over the last $600 \mathrm{~h}$ from $275^{\circ} \mathrm{C}$ up to $294^{\circ} \mathrm{C}$. The higher temperature and the temperature profile were made possible by using current heating of the cable bundle. The temperature of the cable bundles were controlled during the whole test by embedded thermocouples.

Lower temperatures, especially during the transfer phase to mercury, were neglected in the test in favour to simulate the combination of outgassing and UV/VUV in the operational phase of the MPO. The samples underwent a bake out before test with temperatures up to $200^{\circ} \mathrm{C}$ which is also performed on the flight hardware.

The test temperatures of OSRs, Kapton VDA, and white shield coatings were not controlled. Pre-test results showed temperatures for cycle $1-4$ of $183^{\circ} \mathrm{C}$ for Kapton VDA, $179^{\circ} \mathrm{C}$ for OSRs, and 185 to $189^{\circ} \mathrm{C}$ for the white coating samples. In the pre-test for cycle 5 the Kapton VDA samples showed temperatures around $203^{\circ} \mathrm{C}$, the OSR temperature increased to $197^{\circ} \mathrm{C}$, and the white coatings sample temperature ranged between 204 to $207^{\circ} \mathrm{C}$. The temperature spread of the samples was also monitored during the test with an IR camera. A snapshot during test is shown in Fig. 4.

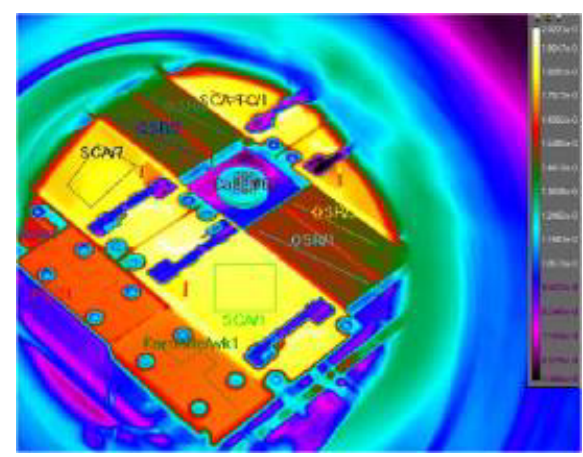

Figure 4. Infra-red camera picture of samples during test exposure

The samples were illuminated with a UV solar aspect angle (SAA) of $0^{\circ}$ and VUV SAA of $45^{\circ}$, whereas in operational flight conditions the MPO solar panel surface will be inclined between a SAA of $71.7^{\circ}$ and $83.8^{\circ}$ [4]. This approximation of UV and VUV irradiation conditions was accepted, however, in order to collect as many equivalent sun hours as possible in a reasonable test time.

The contamination during the test was monitored by witness samples on the sample plate as well as on the facility cold shroud.

The test was split into in total five test runs (cycles) with intermediate inspections after 200,600, 1400, and 2400 hours. The full set of inspections was performed before test and after 3000 test hours. The characterizations of the samples included electrical performance measurement of SCA samples under different angles, and thermo-optical measurements under normal incidence as well as for the thermal control materials under different angles (only OSRs, VDA, and white coatings before test and after 3000 hours). All samples were visually inspected after each test cycle.

\section{RESULTS}

\subsection{Visual}

The visual inspections showed especially on the white coating materials a significantly darkening as can be seen in Fig. 5a. The Kapton VDA is shown in Fig. 5 b and a darkening was also obvious from visual inspection. The SCA samples showed a darkening of the wire insulations and adhesive potter points; however the solar cell and its cover glass respectively did not show an obvious contamination. An example for the SCA samples is depicted in Fig. 5 c. Also on the OSR no obvious contamination layer was observed as can be seen in Fig. 5 d. The cable bundle with Nextel sleeve in Fig. 5 e showed also a pronounced darkening.

a

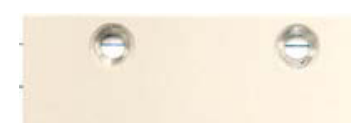

b
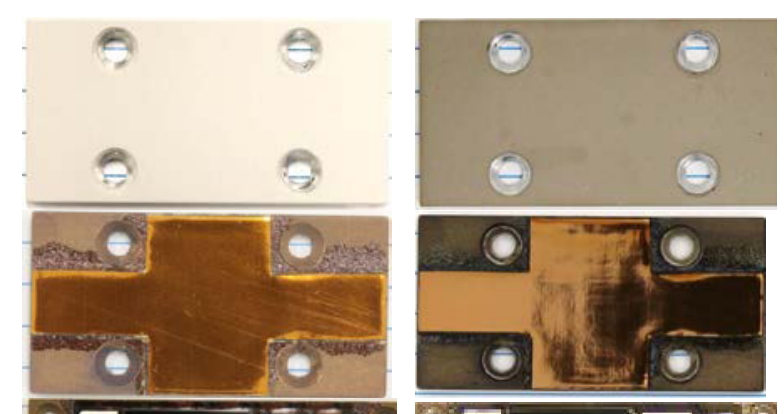

c
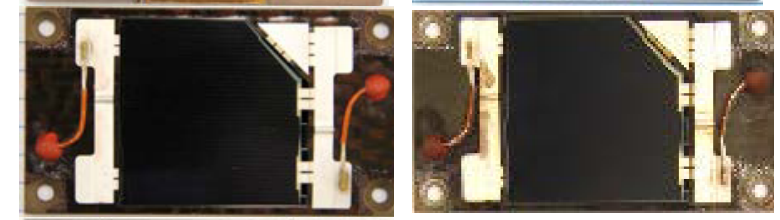

d
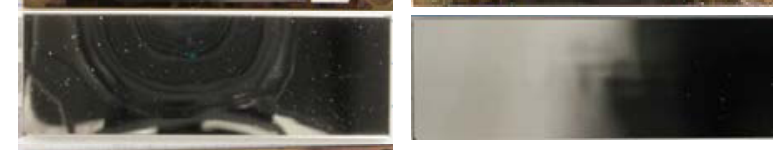

e
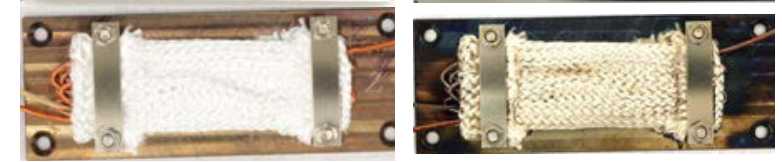

Figure 5.Pristine and exposed (3000 test hours) samples 


\subsection{Solar Cell Performance}

The solar cell current showed a higher degradation in the UV/VUV tests than in UV tests on solar cell assemblies and a test conducted at AM0 [4]. In Figure 6 the resulting difference in short circuit current to begin of test is plotted. The test hours of the UV/VUV test were scaled with a factor of 3.45 to account for the difference in SAA illumination in the other tests with $\mathrm{UV}$ and AM0. There a SAA of $70^{\circ}$ was tested while in the UV/VUV the samples were irradiated by UV with a SAA of $0^{\circ}$ resulting in a higher acceleration.

The lifetime test duration was defined as 6700 hours. The UV tests yielded a degradation of around $-2.5 \%$. In comparison to this value, the interpolated percentage value from the UV/VUV degradation lies at $-6.1 \%$ after this test time. The difference of roughly $4 \%$ can directly be attributed to additional contaminants in combination with the VUV radiation: The VUV radiation is completely absorbed on the SCA surface and does not penetrate deeper, for example to the cover glass adhesive. Therefore there is no reason to attribute the degradation observed to additional degradation in these layers. During the UV test, these layers were exposed to the same UV dose as in this test. In the AM0 test, on the other hand, a similar contamination environment was present, but not the enhancing effect of VUV radiation.

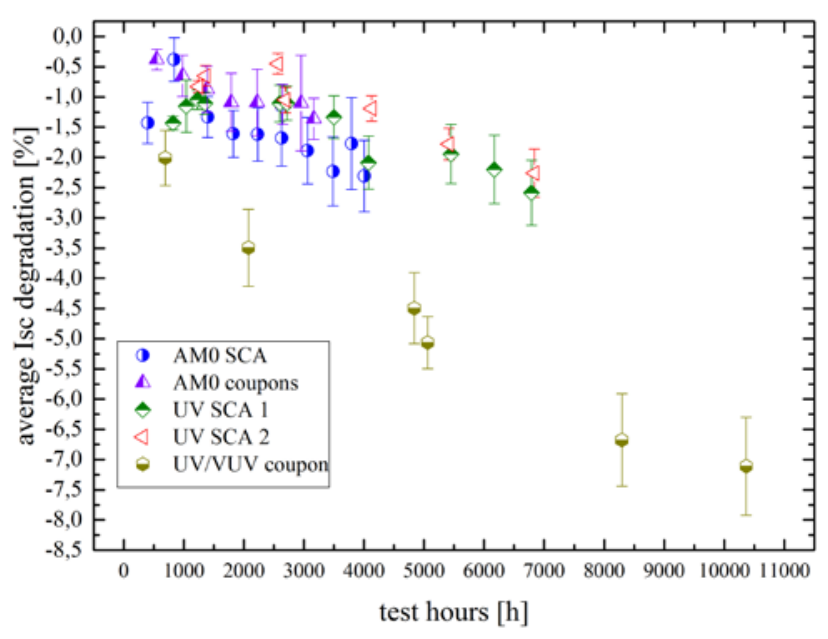

Figure 6. Comparison of Isc degradation in different tests (note: UV/VUV test hours scaled to equivalent $U V$ exposure time under $S A A 70^{\circ}$ )

It is out of the scope of this work to discuss the effect of VUV for photo enhanced contamination and degradation of the contamination layer. In any case the high energetic electromagnetic radiation will cause further photochemical reactions on the molecules that are produced in the outgassing environment.

A consequence of the test results is that an additional contamination loss factor has to be considered in the performance prediction of the solar array for the BepiColombo mission.

\subsection{Thermo-optical Properties}

The SCA samples were also characterized in their thermo-optical properties over test time. However, neither the SCA absorbance nor the emittance values showed an increase.

In general all samples showed a stable emittance over high temperature exposure and UV/VUV irradiation.

The solar absorption values degraded for the thermal control materials over exposure time. The Kapton VDA samples showed an increase in absorption, which is after 3000 test hours still in a linear regime as can be seen in Fig. 7. The increase in solar absorption is most probably a combination of intrinsic degradation of polyimide, which is directly facing the UV/VUV radiation, and a contamination effect. The degradation in the polyimide layer after irradiation is also observed as a darkening on the wire insulation. A quantification of the contribution of the two mechanisms for the increase in alpha is however not feasible, since a comparison with results without VUV and contaminants is not available.

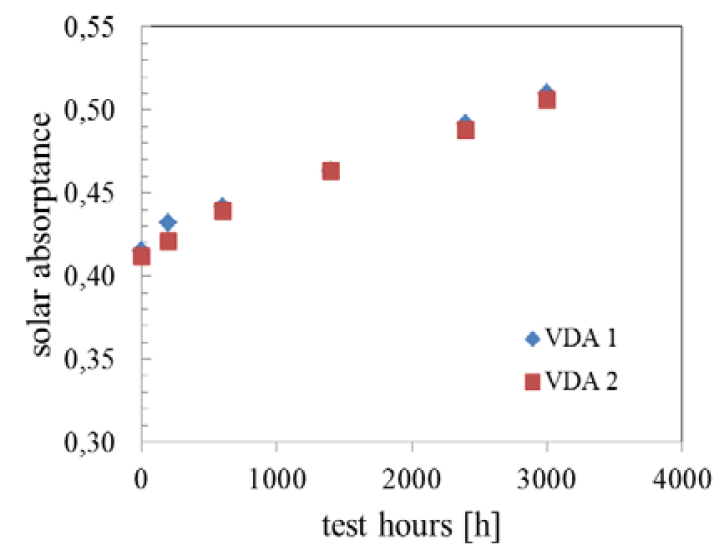

Figure 7. Solar absorptance values of Kapton VDA over test duration

Several white coatings were tested: White Keplacoat and white Keronite (both plasma oxide coatings), and PSZ (plasma sprayed zirconia). The coatings are applied on aluminium. In Fig. 8 the increase in solar absorption of white Keronite is shown. The observed increase is again in line with the visual observation. There seems to be already a trend towards saturation, however, a stable value is not yet achieved after $3000 \mathrm{~h}$. 


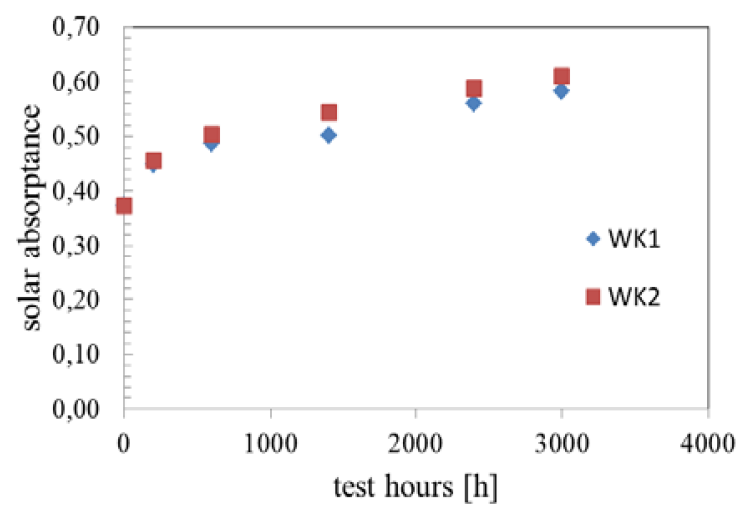

Figure 8. Solar absorptance values of white Keronite samples over test duration

The Nextel sleeve wrapped around a cable bundle darkened visually after exposure. The measurement of the thermo-optical properties yielded no degradation of the emissivity. The solar absorptivity was 0.38 on the exposed sample after 3000 test hours. A pristine sample measured in parallel had a value of 0.05 . A second exposed sample which was used for the pre-test with total duration of approximately 340 hours had a value of 0.21 . In this case it was not feasible to measure the thermo-optical properties at further intermediate test steps because the Nextel sleeve had to be removed from the sample and cut in order to get a flat surface for measurement.

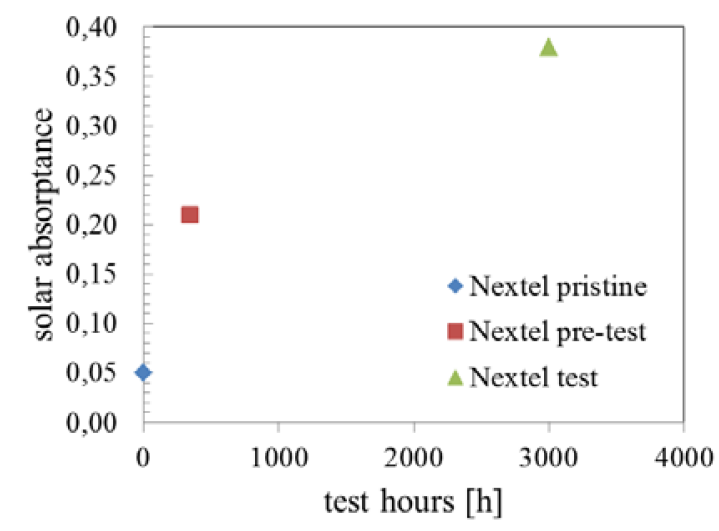

Figure 9. Solar absorptance values of Nextel samples over test duration.

The solar absorption values of the OSR samples show a significant increase over test time. After 3000 test hours the increase has still a linear behaviour. A long term linear increase in absorption was also observed for OSRs during 11 years of the SOHO mission [5]. The increase was suspected to stem from contamination induced degradation.

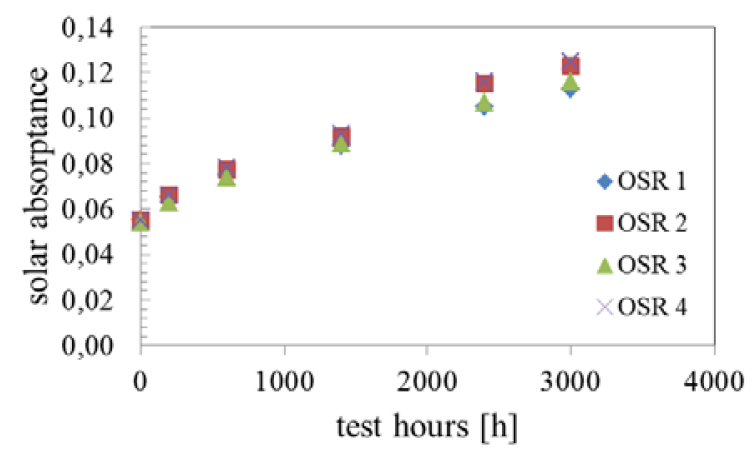

Figure 10. Solar absorptance values of OSRs over test duration

\section{SUMMARY}

A novel test approach was included in the qualification of solar array materials for the high temperature and high intensity mission BepiColombo. The aim of the test was to mimic the contamination environment in combination with the harsh environmental conditions during mission to gain insight into the impact of synergistic effects on the performance of the solar arrays of MPO and MTM.

The results show that significantly different degradation behaviour can be observed in terms of solar cell current degradation. The other cell parameters showed a similar behaviour than in other tests. This can be attributed to a contamination effect that will reduce the light input and thus impacts the cell current only. In comparison to the optical properties the solar cell current is more sensitive to the contamination.

The degradation of the thermo-optical properties can be used to validate input values for thermal analysis. A difficulty that arises in this case is that the alpha values did not saturate within the test time of 3000 hours. For cases where a lifetime is fairly close to the timeframe covered in the test a conservative but ultimately unphysical approach of a linear extrapolation can be used to identify the limitations for the overall thermal analysis. In some cases this extrapolation would lead to very critical consequences. Therefore an extension of the test with part of the samples has been started.

\section{ACKNOWLEDGEMENT}

This work was carried out under a program of and funded by the European Space Agency. The view expressed herein can in no way be taken to reflect the official opinion of the European Space Agency.

The constructive and helpful contributions of Cristina Borrero del Pino, Andreas Löhberg, Yuriy Butenko, Adrian Tighe, Jose Ramon Gonzalez, and Emilio Fernandez Lisbona are gratefully acknowledged. 


\section{REFERENCES}

1. Hall, D.F. et al, Photo-Enhanced Spacecraft Contamination Deposition, AIAA 20th Thermophysics Conference, AIAA-85-0953, 1985.

2. Stewart, T.B. et al, Photochemical Spacecraft SelfContamination: Laboratory Results and Systems Impacts, AIAA Thermophysics, Plasmadynamics and Lasers Conference, AIAA-88-2728, 1988.

3. Löhberg, A. et al, The BepiColombo mercury planetary orbiter (MPO) solar array design, major developments and qualification, this conference

4. Oberhüttinger, C. et al, Simulating large area, high intensity AM0 illumination - test results from BepiColombo and Solar Orbiter qualification, this conference

5. Poinas P., SOHO OSR degradation 11 years of flight data, report TEC-MCT/2008/3635/ln/PP 